\title{
The line of least resistance
}

ACS Energy Lett. 5, 1077-1082 (2020)

Pairing up solar cells into a so-called tandem architecture is an effective strategy to boost the power conversion efficiency. To maximize the gain in efficiency, the photovoltaic parameters of the constituent cells should be specially tailored for tandem application. For instance, the open-circuit voltage and short-circuit current of the tandem cell are directly correlated with those of the individual cells. However, the influence of each sub-cell on the tandem cell's fill factor - the ratio of the actual maximum power to the theoretical maximum power - is less clear and hence more difficult to control. Now, Mathieu Boccard and Christophe Ballif at the École Polytechnique Fédérale de Lausanne in Switzerland show that the fill factor of tandem devices is influenced by shunt resistances rather than series resistances as in single-junction devices.

The researchers simulate the currentvoltage curves of a two-terminal tandem cell using experimental values based on perovskite-silicon devices. By varying the series resistance and shunt resistance, they find that these parameters impact differently on the fill factor of the sub-cells and of the tandem device. In particular, strategies to improve the fill factor in single-junction cells that minimize series resistance have little effect on the fill factor in tandem applications. By comparing two designs for the bottom silicon cell, the researchers suggest that silicon devices that have relatively high series resistances but good open circuit voltage, such as silicon heterojunctions, actually enable higher gains in the tandem device's efficiency than sub-cells with high fill factor.

Giulia Tregnago

Published online: 20 April 2020

https://doi.org/10.1038/s41560-020-0611-z 